Stasiv Iryna. The Use of Compression Sonoelastography in Multiparametric Ultrasound Examination in Cases of Benign Ovarian Formations. Journal of Education, Health and Sport. 2021;11(10):229-241. eISSN 2391-8306. DOI http://dx.doi.org/10.12775/JEHS.2021.11.10.021

https://apcz.umk.pl/JEHS/article/view/JEHS.2021.11.10.021

https://zenodo.org/record/5634861

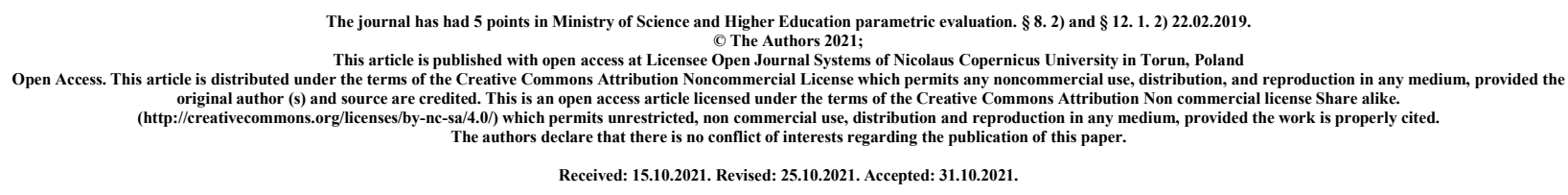

\title{
The Use of Compression Sonoelastography in Multiparametric Ultrasound Examination in Cases of Benign Ovarian Formations
}

\author{
I.D. Stasiv
}

Ivano-Frankivsk National Medical University, The Department of Radiology and Radiation Medicine, Ivano-Frankivsk, Ukraine ORCID ID: 0000-0002-6076-9298, e-mail: irman@meta.ua

\section{Abstract}

Properly diagnosed benign ovarian formations are a condition for appropriate treatment choice. Qualitative assessment of signs detected by multiparametric ultrasound, including compression elastography, is highly effective in the differential diagnosis of benign ovarian formations. Our study became especially relevant for women in the reproductive period since the correct diagnosis influenced the choice of surgical treatment in order to preserve the ovarian reserve. The detailed sonographic aspect of these formations in B-mode, Doppler mode and compression sonoelastography mode is analyzed in the article. Color Flow Mapping, power Doppler and pulsed wave Doppler were used to study blood vessels. Pulsed wave Doppler characterized blood flow quantitatively. Color Flow Mapping was used to determine vessels localization, and power Doppler was used to provide a detailed qualitative assessment of blood flow loci. Elastotype according to the Ueno scale as a qualitative feature and Strain Ratio (coefficient of deformation) as a quantitative indicator were determined for all types of benign ovarian formations. Serous and mucinous cystadenomas were found to belong to elastotypes 0 and I according to the Ueno scale, the papillary component of serous superficial papillomas was mapped as elastotype I and II, fibroids predominantly belonged to elastotype II and III, mature teratoma appertained to elastotypes IV and V. Quantitative coefficient of deformation for all benign ovarian formations ranged from 0.63 to 24.9. According to our results, ultrasound 
examination of ovarian formations is an accurate and highly informative method for stratification of risks in accordance with the O-RADS classification.

Key words: benign ovarian formations, compression sonoelastography, ultrasound examination.

\section{Introduction}

Maintaining the health of the population is a basic requirement for the healthcare industry which lies in a differentiated approach to each patient. Particular attention is drawn to the female population which ensures the preservation of the humankind due to its reproductive function. The preservation of the ovarian reserve is the most important in this aspect in the case of ovarian mass which often cause menstrual, reproductive, sexual dysfunction and disability of women. A large number of publications in national and world literature are devoted to this pathology confirming its importance for science and practice. They present a significant advance in pathogenesis revelation, substantiate the number of existing treatment methods, varieties of which are widely used in clinical gynecology and reproductive medicine with positive consequences in terms of timely and early diagnosis. The search for highly informative and noninvasive diagnostic methods remains a promising direction, which has become a prerequisite for this study. Diagnostic efficiency of compression elastography when differentiating the nature of ovarian neoplasms justifies the feasibility of its inclusion to ultrasound examination methods. Ultrasound compression elastography is proved to provide qualitatively new information about the state of tissue density, which depends on the nature of the lesion. Non-invasiveness, simplicity and quickness peculiar to this method justify its use in the differential diagnosis of ovarian mass, especially at the early stages of the disease.

\section{Justification of Study}

Characterization of ovarian formations and differential diagnosis between benign and malignant formations are important both to reduce unnecessary patients' anxiety and to decide on appropriate treatment choice, which, in turn, optimizes and improves patient's survival and preservation of reproductive function. Benign pathology is treated in a conservative or surgical way in the general gynecological department using minimal access. On the contrary, patients should be referred to specialized oncology departments if malignancy is suspected $[1,2]$.

In recent years, ultrasound diagnostics has been supplemented by a number of new methods based on various physical laws. In particular, the possibility to obtain real-time information about the density of the studied organs using the method of elastography has appeared. Ultrasound examination has reached a qualitatively new level - the so-called ultrasound "palpation" [3]. A deformation of the studied tissue occurs under the action of 
compression providing the information about its elasticity. The obtained data are analyzed by means of an ultrasonic device and an elastography is performed, which reflects the degree of tissues stiffness due to different color spectra and indicates a quality of elastography. A semiquantitative feature is also determined, namely the strain ratio, which is based on the comparative characteristics of the neoplasm and the surrounding tissue. Any neoplasms with high stiffness are associated with an increased risk of malignancy [4,5,6,]. In addition, ovarian reporting and data system (O-RADS) has been established. This is a stratification and ultrasound risk management system designed to provide reliable interpretations, reduce or eliminate ambiguity in ultrasound protocols leading to a more accurate determination of the risk of ovarian malignancy and other formations. It also provides management guidance for each risk category. These recommendations are a common, interdisciplinary, international approach that combines European and North American approaches. The guidance includes all risk categories with their associated management strategies that have not been included in any of the previous systems. $[7,8,9,10]$.

The objective of the research was to determine the diagnostic role of compression sonoelastography in multiparameric ultrasound examination of benign ovarian formations.

\section{Materials and methods}

51 women with benign ovarian formations underwent a comprehensive radiological study. The mean age of patients constituted $37.3 \pm 8.7$ years. All women underwent transvaginal and transabdominal ultrasound examination of the pelvic organs and abdominal cavity. In addition, all patients underwent computed tomography or magnetic resonance imaging. Irrigoscopy was additionally conducted to all patients. Complete blood count, coagulogram and the level of CA125, HE-4, PEA, CA -19-9 cancer-specific markers were necessarily considered among the laboratory methods of examination. The detected ovarian formation was morphologically verified in all cases. The results of the histological conclusion performed after the surgery and laparoscopy were distributed as follows:

- $\quad$ serous cystadenomas - $16(31.38 \%)$;

- $\quad$ serous superficial papillomas - $13(25.49 \%)$;

- mucinous cystadenomas - $9(17.65 \%)$;

- mature teratoma - $8(15.68 \%)$;

- fibroids - $5(9.8 \%)$.

The size of the detected benign ovarian formations in women ranged from $1.9 \mathrm{~cm}$ to 10.5 $\mathrm{cm}$. 
Medical history and past medical history, menstrual and reproductive functions were additionally studied, the results of bimanual gynecological examination and cytology were taken into account.

Ultrasound examination was performed by means of HITACHI ALOKA ARIETTA 70 using cavitary multi-frequency transducer with a frequency of 7.5-10 $\mathrm{mHz}$ and a sector transducer with a frequency of $2-5 \mathrm{mHz}$. Hemodynamic parameters of blood flow were characterized by Color Flow Mapping and power Doppler as well as Pulsed Wave Doppler Mode. The compression elastography mode was used to assess the stiffness of the detected formation. A qualitative characteristic was assigned to the formation according to the UENO stiffness scale, and the determination of the Strain Ratio stiffness index provided a semiquantitative feature of the formation. The study was conducted in real-time mode. The region of interest was denoted as ROI with obligatory optimization of sonoelastography parameters: intensity, mechanical index, control of the study area compression which provided a graph on the monitor screen (Fig. 1). In order to determine the elastography parameters adequately, the area of interest included at least $3 / 4$ of the reference unaltered tissue, as the determination of the stiffness index is based on a comparative analysis of the density of normal and pathologically altered tissues. The elastographic image of the affected ovary was compared with the elastographic image of the contralateral part of the ovary, if any, and in the absence - with the surrounding unaltered tissues. The examination results were stored in the form of static images for further correct interpretation of the obtained data.

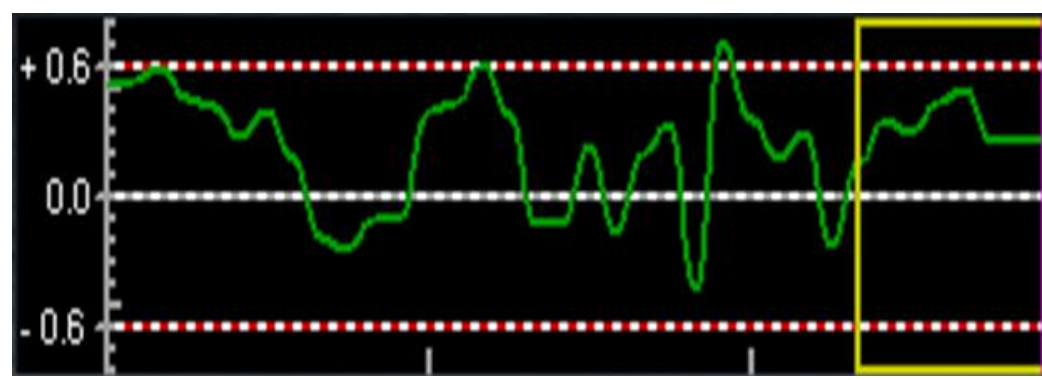

Fig. 1. The scale of compression and pulse recurrence frequency.

Qualitative assessment of neoplasms density was performed using the elastotypes classification according to the UENO scale. Namely, all images that could be attributed to elastotypes 0.1 and 2, corresponded to benign formations. Those belonging to elastotypes 3 were conditionally benign, and images with elastotypes 4 and 5 were characteristic of malignant neoplasms (Fig. 2). 

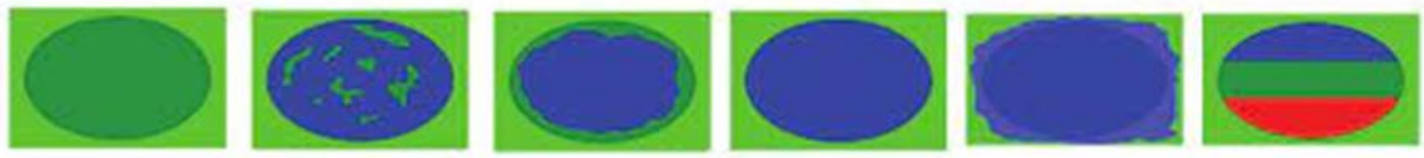

Fig. 2. UENO elasticity scale.

Concurrently, evaluating the qualitative characteristics of the interest focus, a quantitative feature was also assigned to it, i.e. the coefficient of tissues deformation, namely Strain Ratio, was determined. The coefficient was determined by the ratio of ovarian formation density and the density of the surrounding unaltered tissues.

In addition to all the above-mentioned research methods, IOTA ADNEX 2014 mobile application was used which helped to calculate the risk of malignancy. This is a simple calculator where the data of the ultrasound examination, the patient's age, the level of CA-125 are downloaded (Fig. 3).

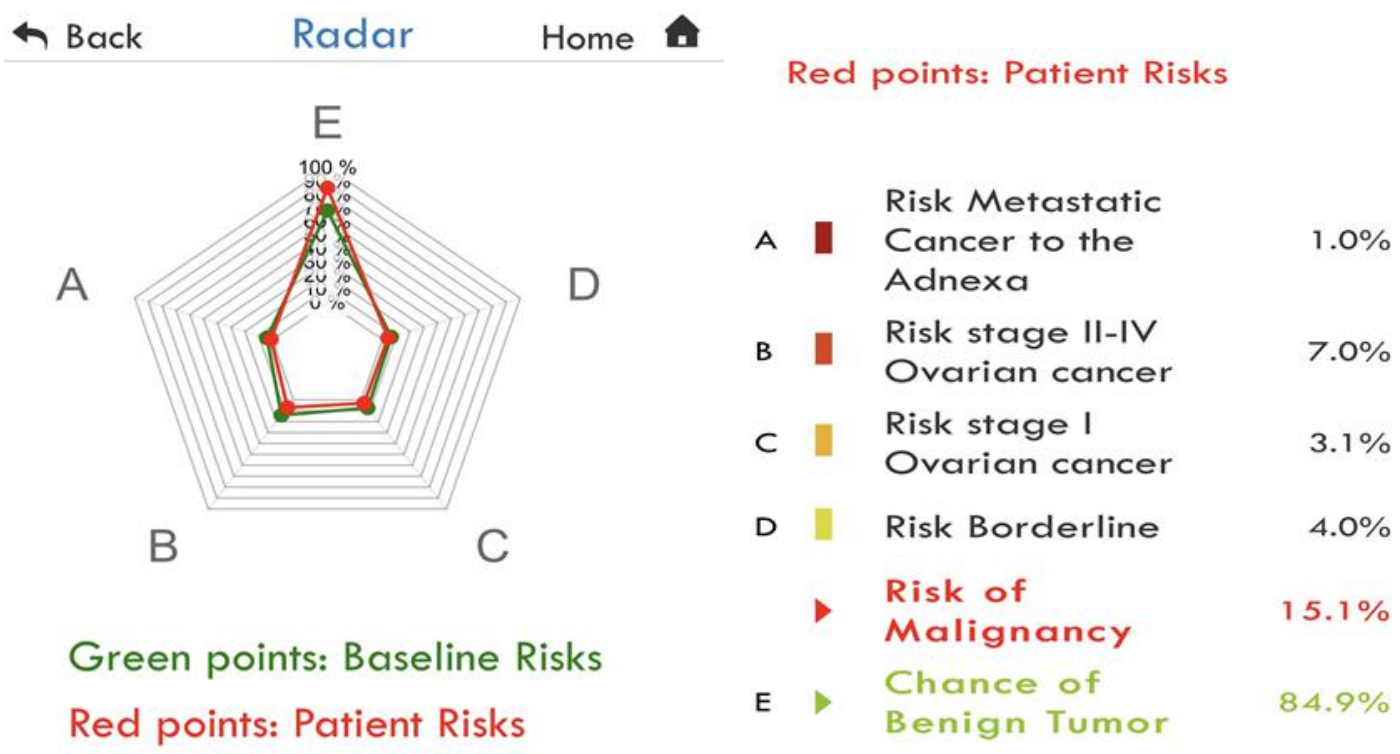

Fig. 3. IOTA ADNEX 2014 mobile application interface.

For the stratification and ultrasound risk management system according to O-RADS system, we followed a consensus guidance from the American College of Radiology. The guidance reduces or eliminates ambiguity in the interpretation of data in ultrasound protocols and provides a more accurate definition of ovarian malignancy. The O-RADS working group includes 5 categories: O-RADS 0 - incomplete evaluation, O-RADS 1 - normal premenopausal ovary, O-RADS 2 -almost certainly benign category ( $<1 \%$ risk of malignancy); O-RADS $3-$ 
lesions with low risk of malignancy ( $1 \%$ to $<10 \%)$; O-RADS $4-$ lesions with intermediate risk of malignancy $(10 \%$ to $<50 \%)$, O-RADS 5 - lesions with high risk of malignancy $(\geq 50 \%)$.

Criteria for inclusion in our studies were the informed consent for ultrasound examination, the presence of benign ovarian formations and the absence of other types of additional ovarian formations.

Exclusion criteria in this group of patients were: patients under 18, pregnant women, patients with acute gynecological pathology.

The obtained research results were processed using the methods of variation statistics. The values of the arithmetic mean (M), mean-square deviation $(\sigma)$ were calculated, the level of probability of differences (p) was determined, the compared group averages were determined using the Student's coefficient ( $\mathrm{t}$ ). Quantitative variables were presented as arithmetic mean (M) \pm arithmetic mean error (m); minimum and maximum values were also indicated.

\section{Results of the research}

Characteristics of ultrasound signs detected by multiparametric ultrasound examination provided in most cases an opportunity to verify individual nosological forms of tumors at the previous stage, which preceded the final morphological diagnosis. This was especially relevant to mature teratomas, serous cystadenomas and fibroids.

Sonographic signs of serous cystadenomas were anechogenic, thin-walled formations with homogeneous content, with average size constituting $4.8 \pm 1.3 \mathrm{~cm}$. The capsule was smooth, regular in all detected cystadenomas. Extranodular blood supply was observed.

The presence of papillary growths with smooth contours on the inner surface of the capsule, with single loci of blood supply was a characteristic sonographic sign of papillary cystadenomas (Fig. 4).

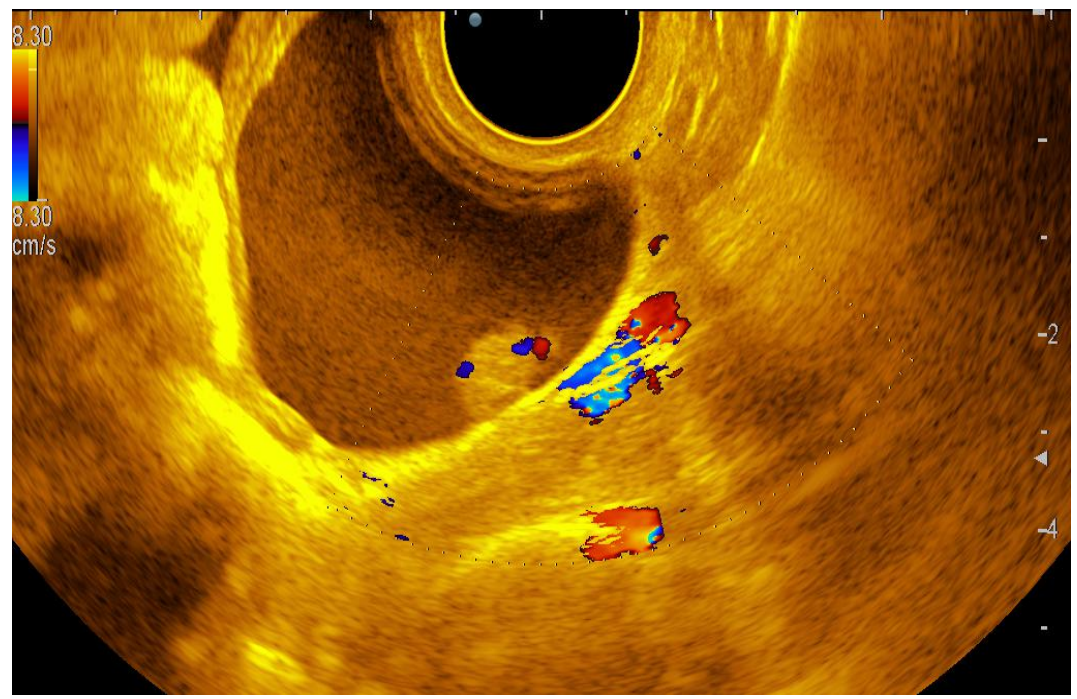

Fig. 4. Sonogram of papillary cystadenoma with color Doppler. 
The average size of mature teratomas constituted $3.2 \pm 0.9 \mathrm{~cm}$. The following features were determined sonographically: heterogeneous, thick-walled formation, with an uneven internal contour, with a parietal hyperechoic component, the so-called "dermoid hump" causing the growth of the internal contents of the tumor, peripheral blood supply (Fig. 5).

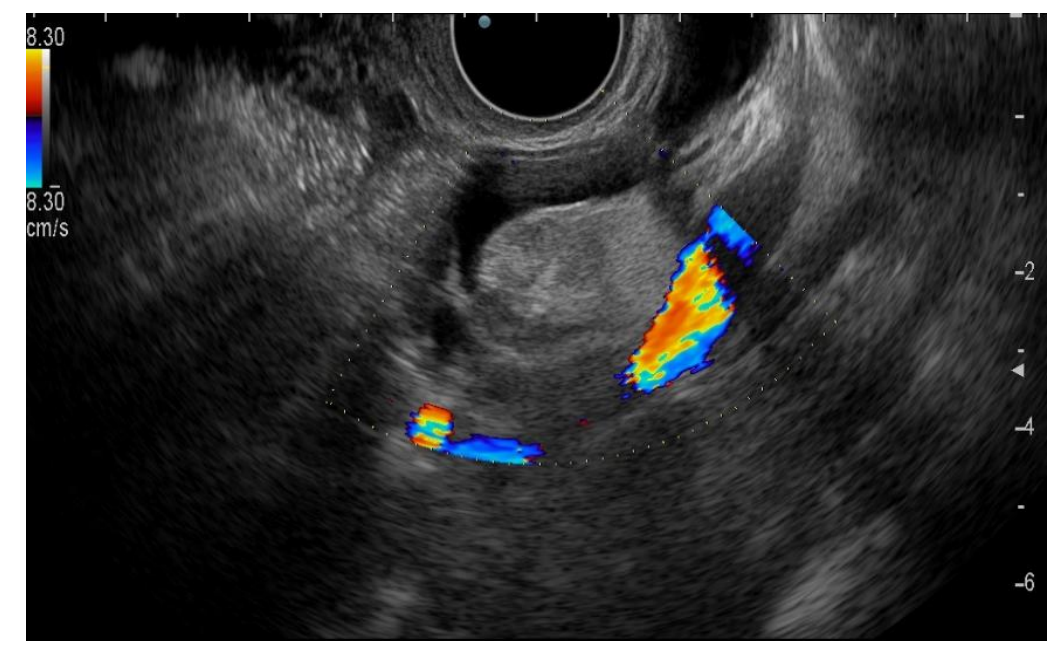

Fig. 5. Sonogram of a mature teratoma with color Doppler.

Benign ovarian formations in $38(74.5 \%)$ of 51 patients were mapped elastically or moderately elastically in the compression elastography mode, stained mainly in green corresponding to elastotype 0 (BGR), 1, 2 and 3 according to the UENO scale. In case of serous cystadenoma, 0 (BGR) elastogram type was peculiar to follicular, paraovarian and endometrioid cysts with mapping of the fluid component in three colors - blue, green and red, and the capsule was mapped in green and red. However, this type of formation was not eliminated up to 6 months of case follow-up, therefore surgical treatment was recommended to such patients. It was also noted that the older the cyst, the more of blue color was observed in the compression elastography mode (Fig. 6). 


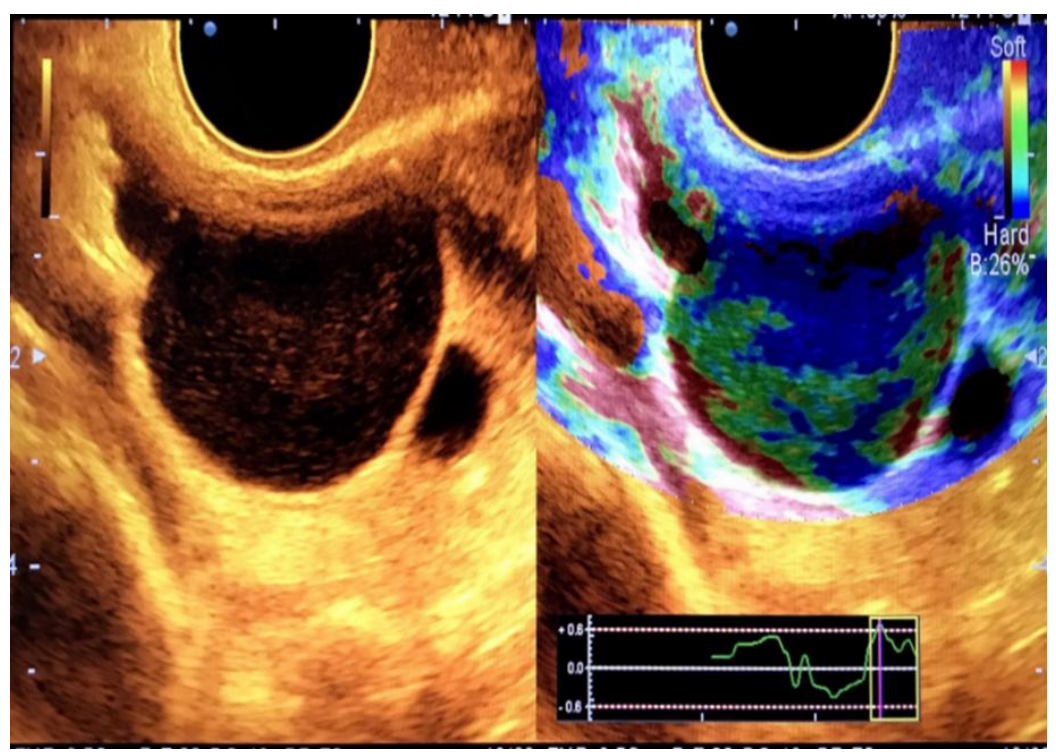

Fig. 6. Sonoelastogram of serous cystadenoma. A-B-mode, B-mode of compression elastography.

Serous superficial papillomas had characteristic signs of benign ovarian formations in 7 (53.8\%) of 13 patients in B-mode using Doppler mode. However, the qualitative assessment of compression elastography increased the method sensitivity. Elastic elastograms with mapping of the solid component and green septa with small impurities of blue corresponding to elastotype 1 and 2 were characteristic for $11(84.6 \%)$ of 13 patients with serous superficial papillomas (Fig. 7).

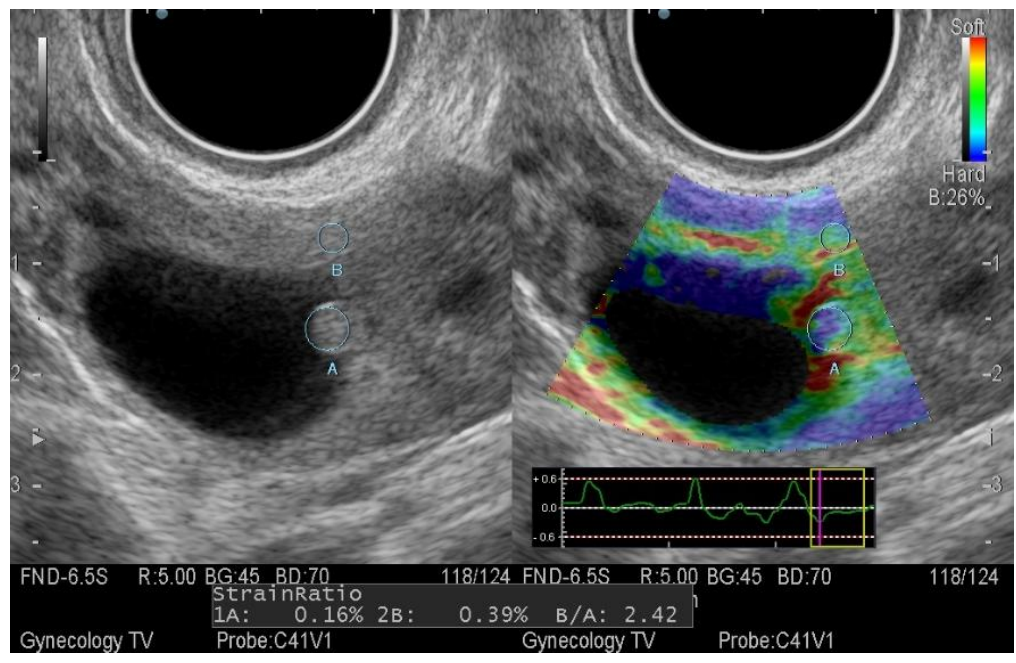

Fig. 7. Sonoelastogram of serous superficial papilloma. A-B-mode, B-mode of compression elastography.

Mucinous cystadenomas of the ovary in all 9 cases were mapped primarily in green, but with tincture of blue and small areas of red corresponding to elastotype 2. This type of 
elastogram was caused by the presence of cystic cavities and the alternation of dense and elastic areas. The walls were elastic in all cases and mapped in green.

Among benign ovarian tumors, special attention should be paid to mature teratomas and fibroids since they have many signs of malignancy, and only a multiparametric approach provides an opportunity to make a more accurate diagnosis before the surgery and to choose the proper method of surgical treatment of such patients.

Fibroids of 5 patients corresponded to elastotype IV and V according to the UENO scale in the compression sonoelastography mode. According to histological data, fibroids contain a fibrous stroma of dense consistency with collagen fibers with polypoid stromal solid growths. This, in fact, influenced the formation of a dense type of elastogram. The false-negative diagnosis was made in 3 cases out of 5 fibroids (Fig. 8).

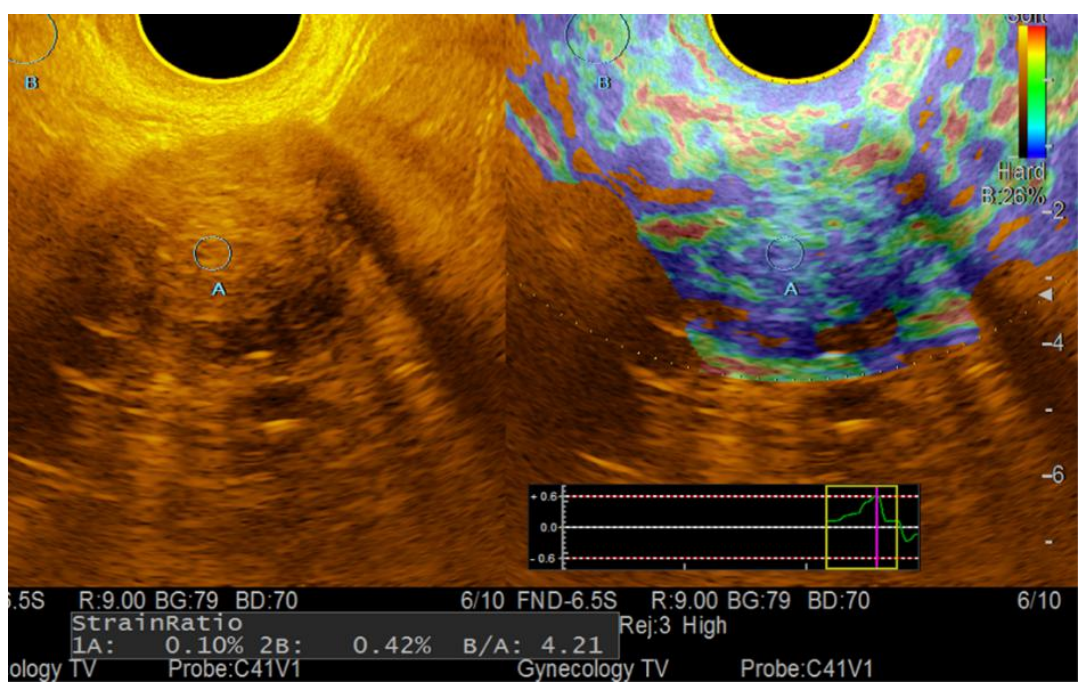

Fig. 8. Sonoelastogram of ovarian fibroids. A-B-mode, B-mode of compression sonoelastography.

Mature teratomas corresponded to elastotype IV in $3(37.5 \%)$ of 8 observations and to elastotype V in $5(62.5 \%)$ cases according to the UENO scale. The high density of teratomas was explained by the predominance of dense inclusions in them such as hair, elements of the dermis, bones, adipose tissue. Classical ultrasound signs of these tumors provided an opportunity to make a correct diagnosis at the stage of research in B-mode and color Doppler mapping. Compression sonoelastography mode presented only additional information about the elasticity and confirmed the high density of this formation (Fig. 9). 


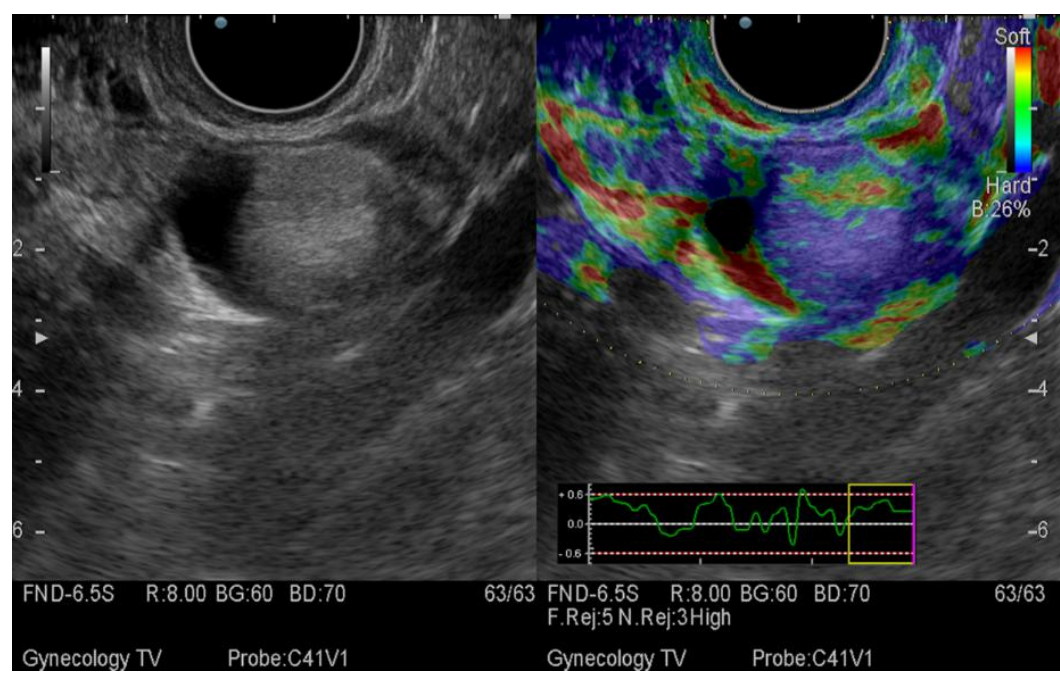

Fig. 9. Sonoelastogram of dermoid cyst of ovary. A-B-mode, B - compression sonoelastography mode.

The only qualitative criterion is not enough for the detection of ovarian formation due to the subjectivity of the compression elastography assessment. Therefore, the density index Strain Ratio was additionally used in all cases of the study. It allowed to quantify the degree of deformation in relation to changes in the density of formation and deformation of the unaltered tissue of the surrounding structures. Determining the stiffness index in benign ovarian tumors, it should be noted that the threshold values for each nosological unit were radically different. Since most benign formations have a cystic structure, and such formations as fibroids and mature teratomas have a predominantly solid structure, the stiffness index will accordingly depend on the predominant component in the formation. Quantitative indicators of compression elastography corresponded to the qualitative indicators of this method, detecting denser ovarian tumors such as fibroids and mature teratomas. Thus, the mean index of stiffness constituted 0.92 \pm 0.46 in serous cystadenomas and $16.7 \pm 8.4$ in mature teratomas. Comparing with other benign formations, the increased density of mature teratomas could be explained by the presence of such elements as fibroblasts, bundles of spindle-shaped cells and bundles of collagen fibers (fibroids), bone tissue and cartilaginous tissue (mature teratoma) in their structure. A low risk of malignancy was determined when analyzing such formations in IOTA ADNEX 2014 mobile application. Considering all ultrasonic descriptors, such as the presence of formations with a solid component with single loci of blood supply, allowed referring these formations to 2 or 3 categories according to the O-RADS classification.

\section{Discussion of the results}

The frequency of serous cystadenoma constituted $31.38 \%$, serous superficial papillomas $25.49 \%$, mucinous cystadenoma $-17.65 \%$, mature teratomas $-15.68 \%$, fibroma $-9.8 \%$ in the structure of benign ovarian tumors. 
Since the successful treatment and preservation of patients' reproductive function depends on the correct management of each woman in particular, the maximum doctor's effort should be aimed at timely and highly informative diagnosis of the detected pathology. In recent years, a growing number of scientists are working to discover new ultrasound descriptors of benign ovarian tumors. Innovative technology of sonoelastography provides qualitatively new information about the tissue elasticity and allows assessing the stiffness of ovarian tumors with high accuracy and specificity. V.Ye. Gazhonova, S.O. Churkina, Ye.B. Savinova [7] note that the sensitivity of the ultrasound method with the use of compression elastography is much higher than with conventional gray scale sonography. However, only a qualitative feature of sonoelastography was determined in their work. It should also be noted that there are no literature data on the value of Strain Ratio for the differential diagnosis of ovarian mass lesions in Ukraine. However, there are Ueno publications with high rates of the method informativeness (sensitivity - 89.4\%, specificity - 88.8\%, accuracy - 89\%) for breast formations at a threshold value of SR 4.3. According to the work by Khalmukhamedova AE [11], a reliable sign of malignant ovarian tumors was the stiffness index constituting more than 5.9, and benign tumors were less than 5.9, accordingly. The difference in our work is that we have failed to establish a specific threshold value of the stiffness index for benign formations, since different nosological units of these ovarian formations have significant differences in stiffness. Therefore, a logical approach in this indicator determination will be its characteristics for each type of benign ovarian formation.

\section{Conclusions}

1. Ultrasound examination as the least invasive method of diagnosis provides an opportunity to diagnose accurately benign ovarian tumors.

2. Since benign ovarian tumors most often occur in the active fertile period of a woman's life, the maximum efforts of gynecologists and reproductive endocrinologist should be aimed at timely diagnosis and treatment of pathology, which will help preserve reproductive potential and reduce the percentage of unnecessary surgery.

3. Innovative technology of sonoelastography provides qualitatively new information about the tissues elasticity and allows assessing the stiffness of ovarian tumors. The stiffness coefficient depends on the nature of the contents and is generally high in case of fibroids and mature teratomas and does not exceed 2.3 in case of serous and mucinous cystadenomas.

4. Compression sonoelastography may be recommended as the part of the algorithm of complex ultrasound examination of the ovaries since it facilitates the stratification and 
management system of ultrasound risks according to the O-RADS system in the risk prediction model.

5. A multiparametric approach to ultrasound diagnosis is a key point in the identification of benign ovarian tumors, taking into account compression sonoelastography.

\section{References}

1. Ionescu CA, Matei A, Navolan D, Dimitriu M, Bohaltea R, Neacsu A, Ilinca C, Ples L. Correlation of ultrasound features and the Risk of Ovarian Malignancy Algorithm score for different histopathological subtypes of benign adnexal masses. Medicine (Baltimore). 2018 Aug; 97(31):11762. https://doi:10.1097/MD.0000000000011762 PMID: 30075600; PMCID: PMC6081138.

2. Mathieu KB, Bedi DG, Thrower SL, Qayyum A, Bast RC. Screening for ovarian cancer: imaging challenges and opportunities for improvement. Ultrasound Obstet Gynecol. 2018; 51(3): 293-303.

3. Egunova MA, Kutsenko IG. Differential diagnosis of benign and malignant neoplasms of the ovaries (history of the issue). Journal of Obstetrics and Women's Diseases.2016. T. 65. No. 6. S. 68-78. https://doi:10.17816/JOWD65668-78

4. Choi JI, Park SB, Han BH, Kim YH, Lee YH, Park HJ, et al. . Imaging features of complex solid and multicystic ovarian lesions: proposed algorithm for differential diagnosis. Clin Imaging. 2016; 40: 46-56. https://doi:10.1016/j.clinimag.2015.06.008 [PubMed] [CrossRef] [Google Scholar]

5. Chekalova MA, Borisova MI. Experience of using compression elastography and contrast-enhanced ultrasound examination in differential diagnosis of primary and metastatic ovarian tumors in patients with uterine cancer. Tumors of the female reproductive system. 2019; 15 (3): 14-23. https://doi.org/10.17650/1994-4098-2019-15$\underline{3-14-23}$

6. Lavrik G, Golovko T, Shevchuk L, Bakai O. Possibility of exchange methods in diagnostics of malignant egg fluff. Clinical Oncology. 2019; T 9, No. 1 (33): 34-41.

7. Gazhonova VE. A new system for the standard of ultrasound examination of ovarian formations for predicting the risk of tumor malignancy. Obstetrics and gynecology. 2020; 10: 28-40 https://dx.doi.org/10.18565/aig.2020.10.28-40

8. Glanc P, Benacerraf B, Bourne T, Brown D, Coleman BG, Crum C et al. First international consensus reporton adnexal masses management recommendations. J. Ultrasound Med. 2017; 36(5): 849-63. https://dx.doi.org/10.1002/jum.14197 
9. American College of Obstetricians and Gynecologists' Committee on Practice BulletinsGynecology. Practice Bulletin No. 174 Summary: evaluation and management of adnexal $\begin{array}{llll}\text { masses. } & \text { Obstet. } & \text { Gynecol. }\end{array}$ https://dx.doi.org/10.1097/AOG.0000000000001763

10. Jacobs IJ, Menon U, Ryan A, Gentry-Maharaj A, Burnell M, Kalsi JK et al. Ovarian cancer screening and mortality in the UK collaborative trial of ovarian cancer sreening (UKCTOCS): a randomized controlled trial. Obstet.Gynecol.Surv.2016;71(6):346. https://dx.doi.org/10.1097/01.ogx.0000483045.61136.eb.

11.Khalmukhamedova AE. Optimization of the algorithm for diagnosing ovarian tumors using ultrasound elastography. Kremlin medicine. 2017; 3: 52-63. 